\title{
INFLUENCE OF SHEAR CONNECTORS ON THE CONCRETE BEAM-COMPOSITE SLAB CONNECTION
}

\author{
Mercy Barroso B., Christian Medina R. y Santiago Medina R. \\ Facultad de Ingeniería Civil y Mecánica. Universidad Técnica de Ambato. \\ mercy.barroso29@hotmail.com; cd.medina@uta.edu.ec; wsmedina@uta.edu.ec
}

Received: June 2017. Accepted: September 2017

\begin{abstract}
In the present work, there were made composite section prototypes with different shear connectors, selected based on the properties of each connector, absorption capacity and shear stress transfer between the metal steel deck and the concrete beam. The connectors that were part of this research include $\varnothing 8 \mathrm{~mm}$ corrugated rod type, steel bolt, steel stud and stirrup type, which are included in the composite system to prevent horizontal and vertical sliding between composite slab and beam, ensure the adhesion with the concrete and provide the beam the maximum resistance to bending.

These specimens were subjected to a bending test to analyze the influence of the shear connectors on the strength of the composite section. Notice that for the element to work as a composite section, it must guarantee the correct interaction between the steel deck and the concrete beam working as a single element. For this, it is analyzed the rupture modulus of each specimen and the moment at the point of rupture of the section is compared with the theoretically calculated moment. Because of the investigation it was determined that the performance of the composite section depends not only on the type of connector but also on the number and spacing of the shear connectors.
\end{abstract}

Key words: Shear connectors, Metal Steel Deck, Slab, Shear Stress.

\section{INFLUENCIA DE LOS CONECTORES DE CORTANTE EN LA UNIÓN VIGA DE HORMIGÓN Y LOSA COMPUESTA}

\begin{abstract}
RESUMEN
En el presente trabajo de investigación se elaboraron prototipos de sección compuesta con diferentes conectores de cortante, seleccionados en base a las propiedades de cada conector, capacidad de absorción y transferencia de esfuerzos cortantes entre la losa con placa colaborante y la viga de hormigón. Los conectores que fueron parte de esta investigación incluyen tipo arco de varilla corrugada $\varnothing 8 \mathrm{~mm}$, tornillo, espigo y tipo estribo, los cuales son contenidos en el sistema para cumplir con funciones como
\end{abstract}


impedir el deslizamiento horizontal y vertical entre losa compuesta y la viga, garantizar la adherencia con el hormigón y brindar la resistencia máxima a la flexión la viga.

Estos especímenes fueron sometidos a ensayo de flexión con la finalidad de analizar la influencia de los conectores de corte en la resistencia de la sección compuesta. Cabe recalcar que para que el elemento trabaje como compuesto, éste debe garantizar la correcta interacción entre la placa de acero y la viga de hormigón trabajando como un solo elemento. Para ello se analiza el módulo de rotura de cada espécimen y se compara el momento en el punto de rotura de la sección con el momento teóricamente calculado. Como resultado de la investigación se determinó que el desempeño de los elementos compuestos no solo depende del tipo de conector sino también del número y espaciamiento de los conectores.

Palabras Claves: Conectores de Cortante, Losa Compuesta, Esfuerzos de Corte.

\section{INTRODUCCIÓN}

En la sección I se presenta una introducción del estado del arte en cuanto a conectores de cortante en losas compuestas. En la sección II se describen tanto el marco teórico como la metodología utilizada para llevar a cabo la presente investigación. En la sección III se analizan y discuten los resultados obtenidos del cálculo teórico, así como los resultados experimentales de los ensayos realizados. Finalmente, las conclusiones más relevantes son presentadas en la sección VI.

La construcción compuesta empezó a utilizarse en 1950, adoptada por diseñadores y constructores de puentes y edificios con el fin de aprovechar las propiedades del acero debido a su resistencia a la tensión y complementarla con el excelente comportamiento ante esfuerzos a compresión proporcionado por el hormigón. Su principal crecimiento en la construcción se dio durante la década de los 60 's como resultado de las disposiciones sobre diseño simplificado introducidas en la Especificación AISC en 1961 (Ollgaard, Slutter, \& Fisher, 1971). El auge de la utilización del sistema compuesto radica en el hecho de que las secciones compuestas por vigas de acero y losa de hormigón incrementan la capacidad de carga del sistema entre $20 \%$ y $30 \%$, dotando de mayor rigidez y por ende menor deflexión al sistema compuesto, a más de aprovechar al máximo la sección, pues se logra que losa de hormigón trabaje a compresión en casi toda su altura (McCormack \& Csernak, 2012).

Los elementos compuestos entonces, deben satisfacer las demandas generadas por cargas externas ya sean estas gravitacionales o laterales inducidas por viento y sismo. Por lo tanto, los conectores de cortante son demandados por el cortante horizontal generado en la interfase de la sección compuesta (Hurtado, Molina, \& Linero, 2008), y cumplen con la función principal de transmitir los esfuerzos generados en la losa de compresión hacia las vigas de acero y/o de hormigón que forman parte del sistema estructural principal resistente a carga lateral. Por lo tanto, el diseño de los conectores de cortante es esencial para garantizar el comportamiento como sección compuesta. Es por esta razón, que a través de los años varios autores han estudiado la influencia de los conectores de cortante en la resistencia de la sección compuesta por losa de steel deck rellena de hormigón armado y viga de acero, siendo el conector tipo espigo (stud) el más empleado en la construcción y por ende en investigaciones 
como la realizada por (Zamora \& Molina, 2008).

Sin embargo, se han realizado escasos estudios acerca de la influencia de los conectores de corte en la conexión entre la losa con placa colaborante y las vigas de hormigón, en los casos en el que el sistema principal lo constituyen pórticos de hormigón armado. Es por esta razón que el objetivo general de este estudio es analizar la conexión entre la losa deck y la viga de hormigón empleando varios tipos de conectores de corte a distintos espaciamientos, y su influencia en el trabajo conjunto entre estos dos elementos.

\section{METODOLOGÍA}

El trabajo como sección compuesta se alcanza una vez que el hormigón alcanza su resistencia e interactúa monolíticamente con la placa colaborante. Para el análisis teórico se aplica la teoría de secciones transformadas de donde se obtiene las propiedades de la sección compuesta. En el caso de voladizo, la placa solo funciona solamente como encofrado permanente y debe diseñarse con un refuerzo superior por momento negativo (Acero Deck, 2003). El esquema de la losa con placa colaborante se muestra en la figura 1.

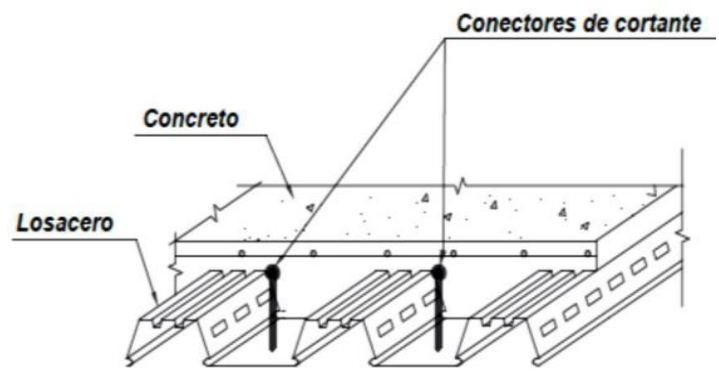

Figura 1 Sistema de Losa Compuesta

Fuente: Construcción compuesta acero - concreto, Gerdau Corsa, (s.f.)

\subsection{Conectores de Cortante}

Los conectores de corte son elementos de acero, cuya función principal es contrarrestar los esfuerzos de corte que se generan en la losa compuesta (lámina de acero - hormigón) controlando y reduciendo las deformaciones además impide la separación vertical entre losa y viga (Acero Deck, 2003). Con ello se logra un mejor aprovechamiento de la altura de entrepiso, además de realizar una unión permanente entre la losa y la viga de apoyo, permitiendo que estos dos elementos trabajen en forma conjunta.

La sección compuesta da como resultado una mayor área resistente a la compresión, permitiendo que se pueda disminuir el peralte de la viga y por lo tanto el costo del sistema, ahorrando hasta un $20 \%$ en peso. En cuanto al diámetro del conector las especificaciones de (AISC 360-10, 2010) establecen un diámetro máximo del conector de cortante de $19 \mathrm{~mm}$, y la cantidad no debe ser mayor a 3 conectores en sentido transversal. 


\subsection{Tipos de Conectores}

Como resultado de investigaciones previas realizadas con diferentes conectores, se tiene que su eficiencia incluye, pero no se limita a variables tales como las características geométricas, material del conector, la resistencia y el módulo de elasticidad del hormigón (Ollgaard, Slutter, \& Fisher, 1971).

Visto de esta forma y dado que las normas establecen que se puede utilizar nuevos tipos de conectores de cortante, siempre y cuando se demuestre su funcionalidad mediante investigaciones, para la presente investigación se utilizaron los conectores enlistados a continuación.

\subsubsection{Conectores tipo Arco}

Este tipo de conector consiste en una varilla de $8 \mathrm{~mm}$, de diámetro doblada en forma de arco la cual se funde monolíticamente con la losa, tal y como se muestra en la figura 2.

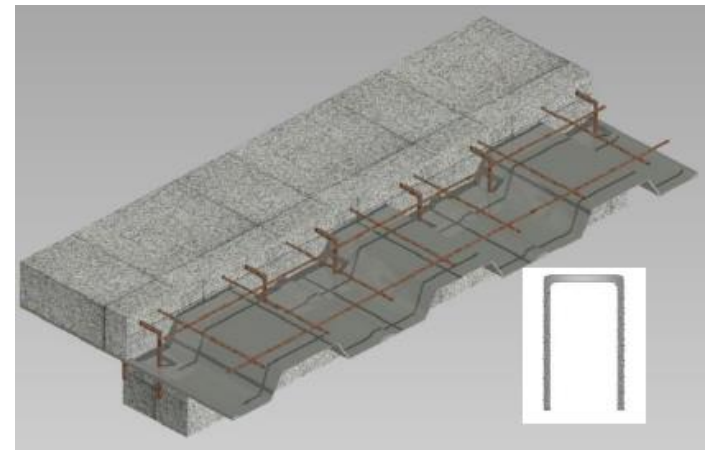

Figura 2 Conector Tipo Arco

Fuente. - Barroso (2016).

Su forma corrugada aporta a una mínima concentración de esfuerzos en el momento en que la fatiga actúa debido a la carga. Estas características hacen apta a la varilla para el uso como conectores de cortante al demostrarse la capacidad de transferir esfuerzo cortante de la losa a la viga de hormigón a través del flujo de corte.

$$
f=\frac{Q * V}{I}
$$

En donde $f$ es el flujo de corte, $V$ es la fuerza interna de corte, $Q$ es el área de la sección transversal del segmento que se conecta a la viga, e $I$ es el momento de inercia de la sección transversal del conector.

\subsubsection{Conectores tipo Tornillo}

El segundo tipo de conectores utilizado en la investigación son del tipo tornillo, los cuales se perforan a través de la placa colaborante para unirla con la viga de hormigón, como se muestra en la figura 3. Este tipo de conector es comúnmente utilizado en la construcción. 


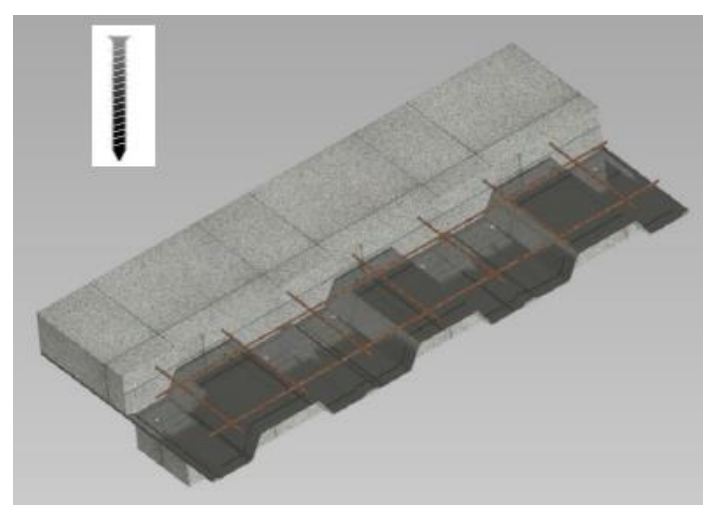

Figura 3 Conector Tipo Tornillo Fuente. - Barroso (2016).

El esfuerzo nominal del conector $Q n$ viene dado por la ecuación:

$$
Q_{n}=0.14 * A_{c p} \sqrt{f^{\prime} c * E_{c}} *\left(\frac{S}{d_{c p}}\right)^{0.25} \leq A_{c p} * F_{u}
$$

En donde $A_{c p}$ es el área de la sección del conector tipo tornillo, $d_{c p}$ es el diámetro nominal del conector, $f^{\prime} c$ es el esfuerzo de compresión del hormigón, $E_{c}$ es el módulo elástico del hormigón $S$ es el espaciamiento entre conectores y $F_{u}$ es el esfuerzo último del acero utilizado para el conector (Acero Deck, 2003).

\subsubsection{Conectores tipo Espigo (Stud)}

Los conectores tipo espigo (stud) son los más utilizados en la construcción los cuales se asemejan a un perno sin rosca, con un vástago y una cabeza, con dimensiones estandarizadas. El esquema de este tipo de conector se muestra en la figura 4.

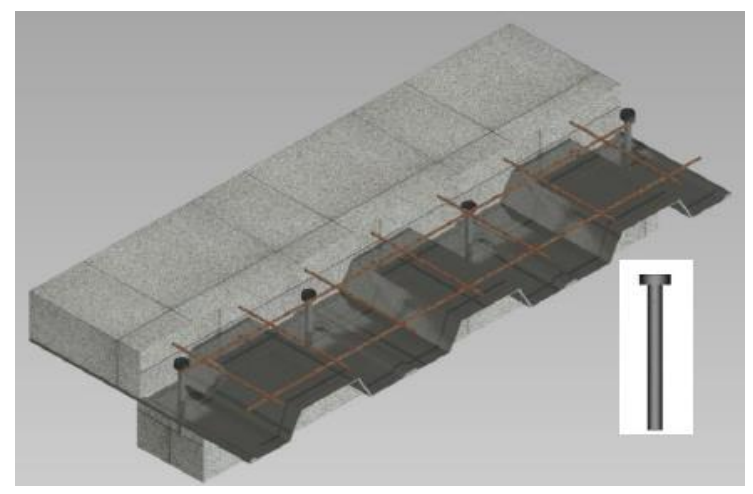

Figura 4 Conector Tipo Espigo Fuente. - Barroso (2016). 
El esfuerzo nominal del conector $Q n$ viene dado por la ecuación:

$$
Q_{n}=0.5 * A_{s c} \sqrt{f^{\prime} c * E_{c}} \leq A_{s c} * F_{u}
$$

En donde $A_{s c}$ es el área de la sección del conector tipo espigo, y el resto de variables ya han sido definidas previamente (Bonilla, Bezerra, Larrúa, Recarey, \& Mirambell, 2015).

\subsubsection{Sección monolítica con los estribos de la viga}

El último tipo de conector corresponde a los propios estribos de la viga de hormigón, para ello en el proceso de fundición de la viga se dejan $10 \mathrm{~cm}$, libres de la altura de la viga, equivalentes a la altura de la losa y en los cuales sobresalen los estribos. Posteriormente se coloca el deck metálico y se procede con el fundido de la losa y la parte restante de la viga. El resultado de la sección monolítica, se muestra en la figura 5.

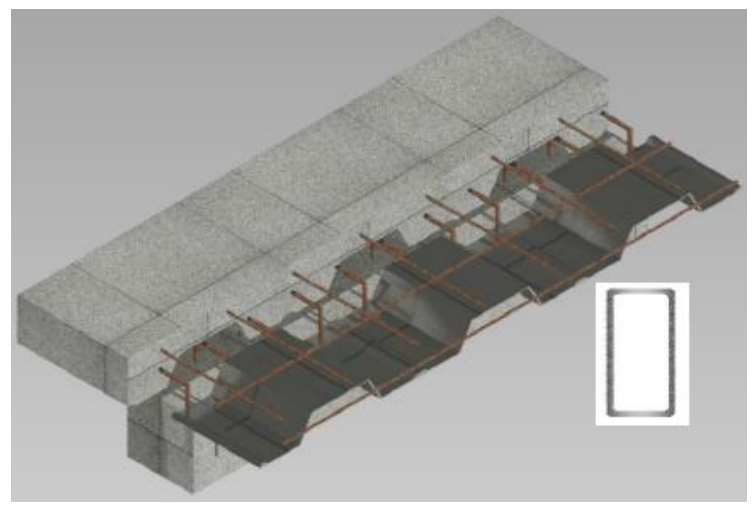

Figura 5 Estribo funcionando como conector. Fuente: Barroso (2016).

\subsection{Espaciamiento de los conectores}

El espaciamiento de los conectores de cortante dependerá de la resistencia al corte del conector y del flujo de corte último o fuerza cortante ultima por unidad de longitud (AISC 360-10, 2010). La fórmula para el cálculo de espaciamiento es la siguiente:

$$
e=\frac{Q t}{S u} \leq 800 \mathrm{~mm}
$$

En donde $Q t$ es la resistencia al corte del conector, Su es la fuerza cortante última por unidad de longitud.

Se procedió con el cálculo del espaciamiento de los conectores de corte, para la elaboración de los prototipos (uno por cada tipo de conector), pero adicionalmente se elaboraron prototipos con conectores de cortante, situados a la mitad del espaciamiento requerido, con la finalidad de evaluar la influencia del espaciamiento en el comportamiento de la sección. Es así que se elaboraron 7 especímenes, 2 con conectores tipo arco, 2 con conectores tipo tornillo, 2 con 
conectores tipo espigo, y 1 en el que los estribos de la viga hacen las veces de conector de corte.

\subsection{Modelo Matemático de la Sección Compuesta}

La metodología incluye la comparación obtenida de los resultados experimentales con los resultados obtenidos de a partir de un análisis teórico. Es así como para el efecto se calculó el momento resistente de la sección a partir de la compatibilidad de esfuerzos y deformaciones de tracción y compresión. Cabe recalcar que, para el cálculo del momento resistente, se consideró el modelo elastoplasto para el acero, es decir no se ha considerado la sobre resistencia post fluencia del mismo, en razón de que se espera que la sección presente otro tipo de fallas (adherencia) mucho antes de que el acero entre en proceso de fluencia. Además, para el caso del concreto, se consideró el modelo del bloque rectangular de Whitney, pues el escaso confinamiento de las varillas longitudinales de la viga hace que la sobre resistencia del hormigón por refuerzo transversal se vea disminuida casi en su totalidad.

Tabla 1 Propiedades de la Sección Compuesta

\begin{tabular}{|l|l|}
\hline PROPIEDAD & VALOR \\
\hline Base viga bv $(\mathrm{cm})$ & 15 \\
\hline altura viga hv $(\mathrm{cm})$ & 15 \\
\hline altura losa hl $(\mathrm{cm})$ & 10.2 \\
\hline Ancho efectivo be $(\mathrm{cm})$ & 50 \\
\hline Recubrimiento $\mathrm{r}(\mathrm{cm})$ & 3 \\
\hline Acero a tracción As1 $\left(\mathrm{cm}^{2}\right)$ & 1.58 \\
\hline Acero a compresión As2 $\left(\mathrm{cm}^{2}\right)$ & 1.58 \\
\hline Acero Deck e=0.65mm As3 $\left(\mathrm{cm}^{2}\right)$ & 0.325 \\
\hline f'c $\left(\mathrm{kg} / \mathrm{cm}^{2}\right)$ & 240 \\
\hline fy $\left(\mathrm{kg} / \mathrm{cm}^{2}\right)$ & 4200 \\
\hline fy deck $\left(\mathrm{kg} / \mathrm{cm}^{2}\right)$ & 2812.23 \\
\hline Es $\left(\mathrm{kg} / \mathrm{cm}^{2}\right)$ & $2.10 \mathrm{E}+06$ \\
\hline Es deck $\left(\mathrm{kg} / \mathrm{cm}^{2}\right)$ & 2070023.91 \\
\hline Ec $\left(\mathrm{kg} / \mathrm{cm}^{2}\right)$ & 188643.19 \\
\hline
\end{tabular}


Estos criterios se han tomado en cuenta para el cálculo teórico de la sección, de manera que el estudio se enfoque en la superficie de interacción entre la placa de acero deck, y la viga de hormigón. Es así como los prototipos responden a los datos indicados en la tabla 1.

Con estos datos se realizó el cálculo del momento resistente de la sección a partir del diagrama de deformaciones y esfuerzos presentados en la figura 6 .

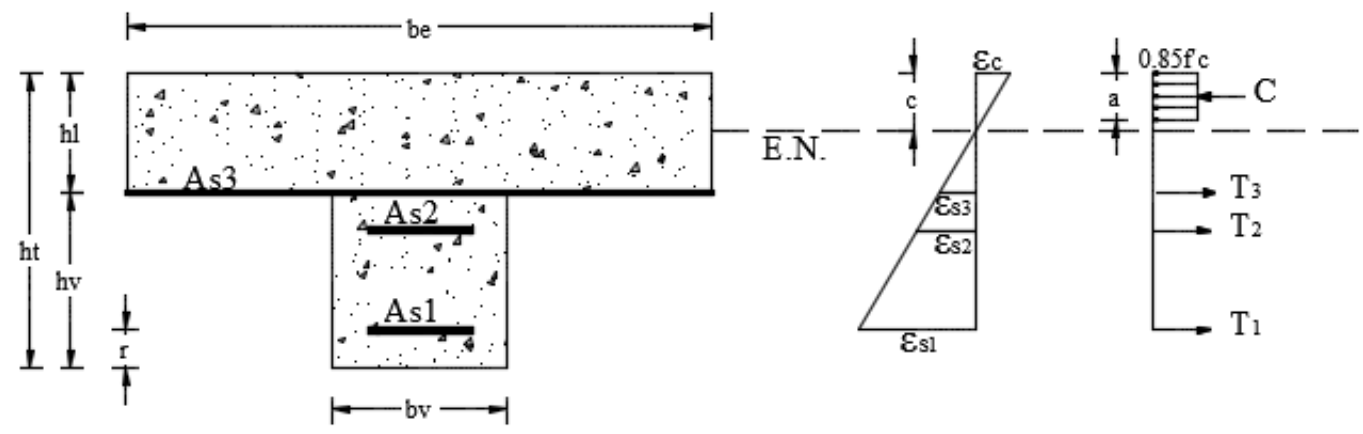

Figura 6 Diagrama de Esfuerzos y Deformaciones Fuente: Barroso (2016).

Es así como el momento resistente de la sección está dado por la ecuación:

$$
M_{n}=T_{1}\left(h t-r-\frac{a}{2}\right)+T_{2}\left(h l+r-\frac{a}{2}\right)+T_{3}\left(h l-\frac{a}{2}\right)
$$

\subsection{Elaboración de Prototipos}

Los prototipos se dimensionaron en función de la capacidad de la máquina de ensayo a flexión, y fueron elaborados en el laboratorio de ensayo de materiales de la Universidad Técnica de Ambato. Una vez elaborados los especímenes se sometieron a ensayo de flexión de acuerdo a la norma (ASTM C293-79, 2016) en los laboratorios de la Pontificia Universidad Católica del Ecuador en Quito. Como se mencionó previamente, se elaboraron 7 especímenes con la misma sección transversal en las cuales se variaron los tipos y cantidad de conectores de corte. 

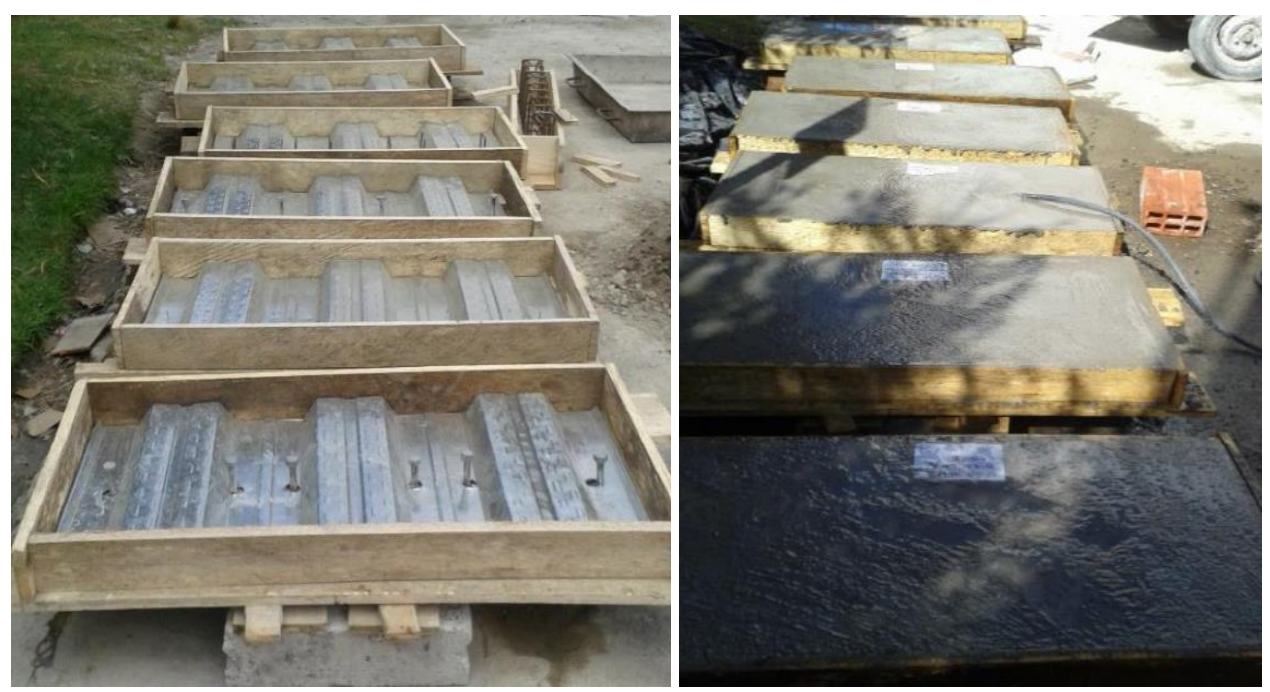

Figura 7 Elaboración de Prototipos a Ensayarse Fuente: Barroso (2016).

En la figura 8 se aprecian las características de los especímenes ensayados.

\begin{tabular}{|c|c|}
\hline LOSA N 1 & 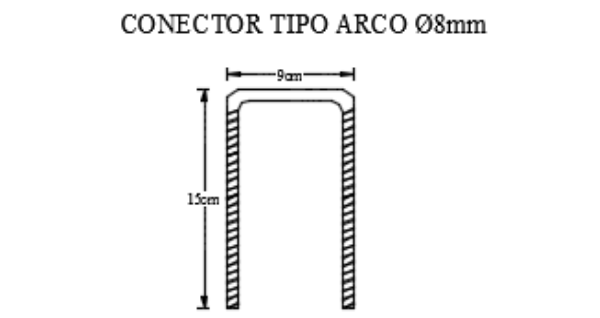 \\
\hline LOSA N$^{\circ} 2$ & $\begin{array}{l}\text { - } \quad \text { Malla electrosoldada de } \emptyset 5.5 \mathrm{~mm} \text { cada } 15 \mathrm{~cm} \text {. } \\
\text { - } \quad \text { Viga de hormigón de } 15 \times 15 \mathrm{~cm} \text {. } \\
\text { - } \quad \text { Altura de losa } 10.2 \mathrm{~cm} \\
\text { - } \quad \text { Conector de cortante tipo varilla corrugada } \\
\text { doblada en arco. } \\
\text { - } \quad \text { Fluencia del acero fy }=4200 \mathrm{~kg} / \mathrm{cm} 2 . \\
\text { - } \quad \text { fc }=240 \mathrm{~kg} / \mathrm{cm} 2\end{array}$ \\
\hline
\end{tabular}

a) 


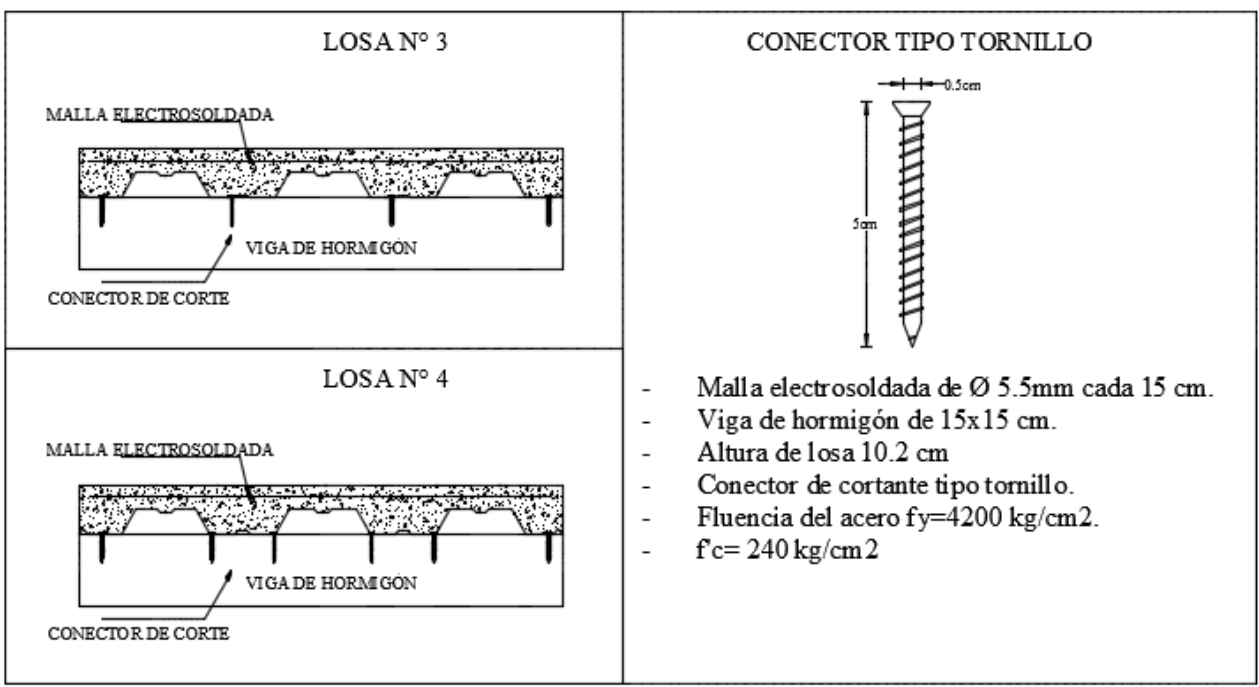

b)

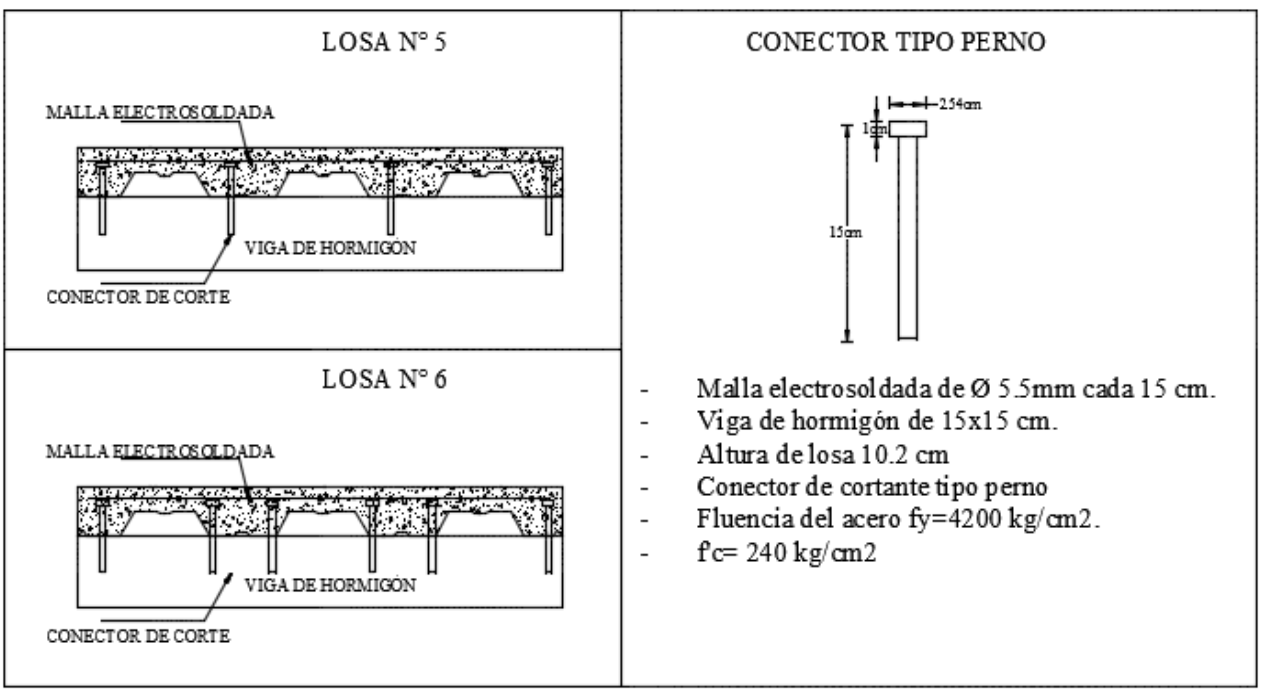

c) 


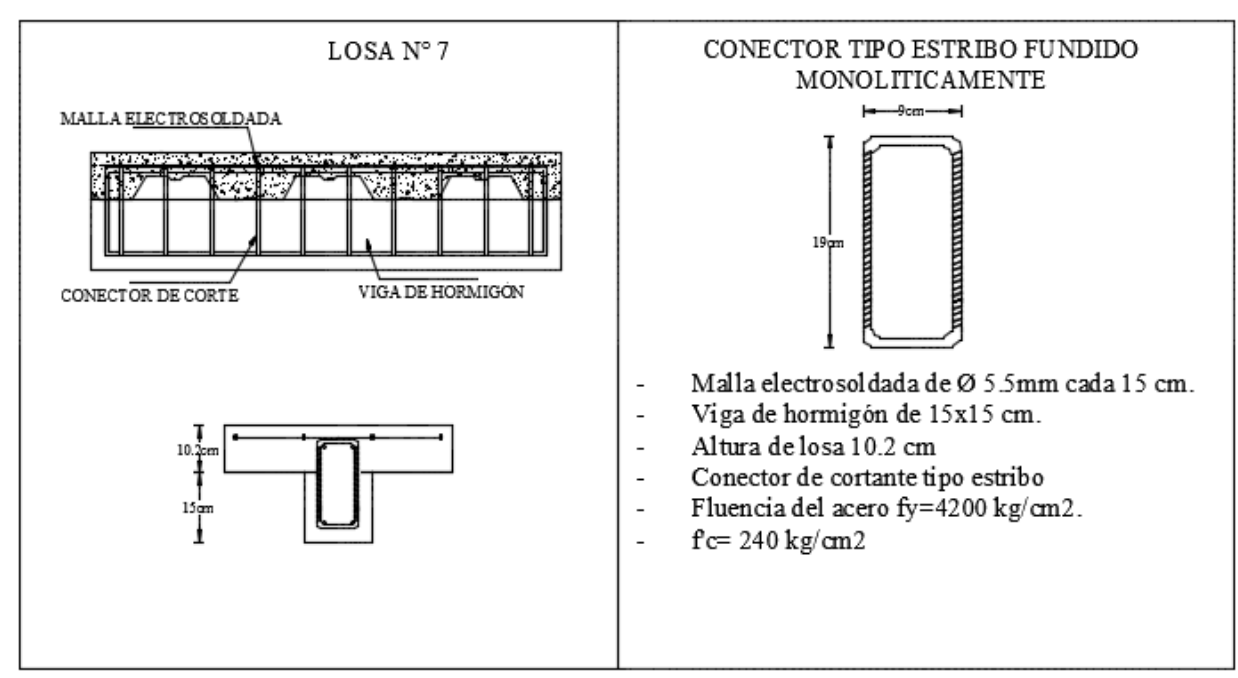

Figura 8 Muestras Elaborados

Fuente: Barroso (2016).

\section{ANÁLISIS DE RESULTADOS}

\subsection{Influencia del Espaciamiento}

Al realizar la comparación entre un mismo tipo de conector de corte a diferente espaciamiento, se observa que para los conectores tipo arco y espigo la separación entre conectores sí influye en la capacidad de carga de la sección, lográndose un incremento de capacidad del 50\% al utilizar mayor cantidad de conectores de corte para el caso de los conectores tipo arco, y del $12.5 \%$ en el caso de los conectores tipo espigo como se lo evidencia en las figuras 9 y 10.

\section{COMPARACIÓN LOSA 1-2 CONECTORES TIPO ARCO Ø8mm}

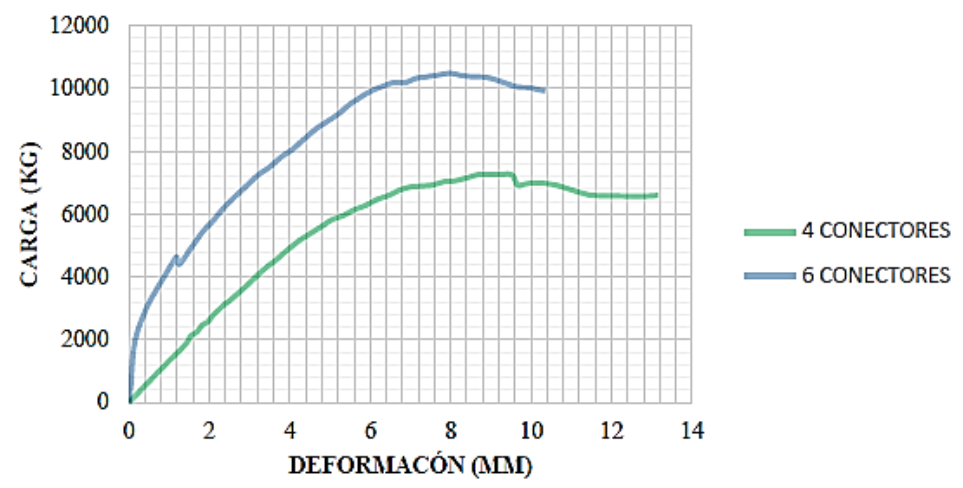

Figura 9 Carga vs Deformación Conector tipo Arco Fuente: Barroso (2016). 


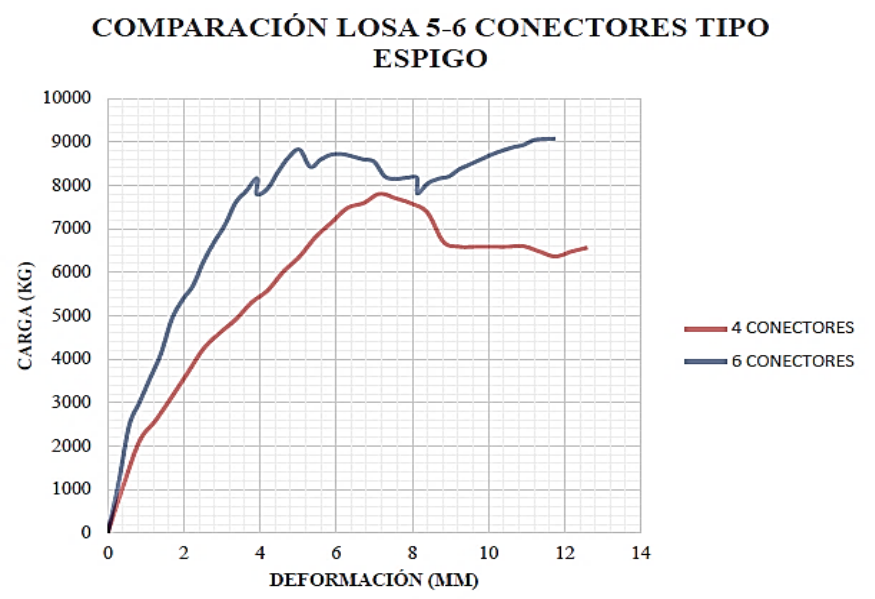

Figura 10 Carga vs Deformación Conector tipo Espigo (Stud) Fuente: Barroso (2016).

Para el caso de los conectores tipo tornillo la capacidad de carga es prácticamente la misma para los dos espaciamientos; esto debido a que este tipo de conector presenta un comportamiento deficiente dando como resultado la menor capacidad de carga de la sección en comparación de las secciones con otro tipo de conectores, como se muestra en la figura 11.

COMPARACIÓN LOSA 3-4 CONECTORES TIPO TORNILLO

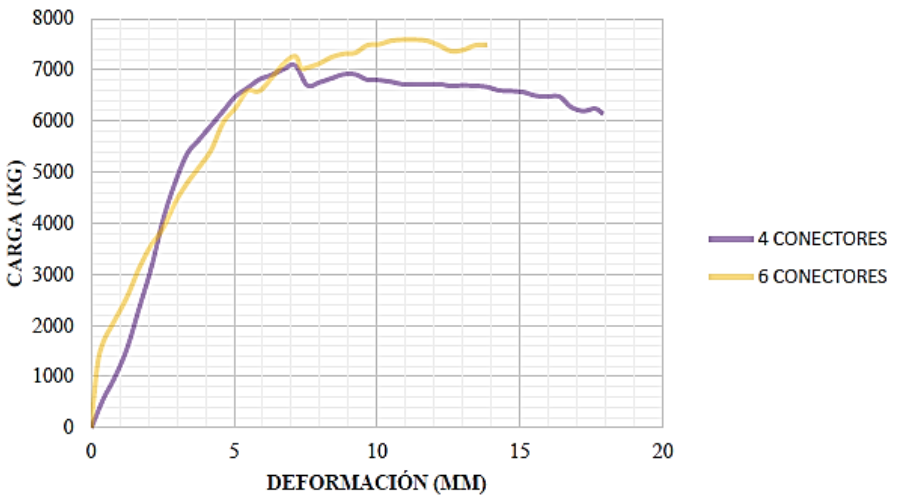

Figura 11 Carga vs Deformación Conector tipo Arco

Fuente: Barroso (2016).

\subsection{Influencia del Tipo de Conector}

La comparación entre conectores se la realiza para los especímenes con 6 conectores, al ser éstos los que alcanzaron mayores valores de capacidad de carga. En los resultados del ensayo de todos los prototipos existe una diferencia en capacidad de carga última del $20 \%$ al $25 \%$, siendo el conector tipo arco el conector 
que mayor capacidad de carga genera, tal y como se muestra en la figura 12.

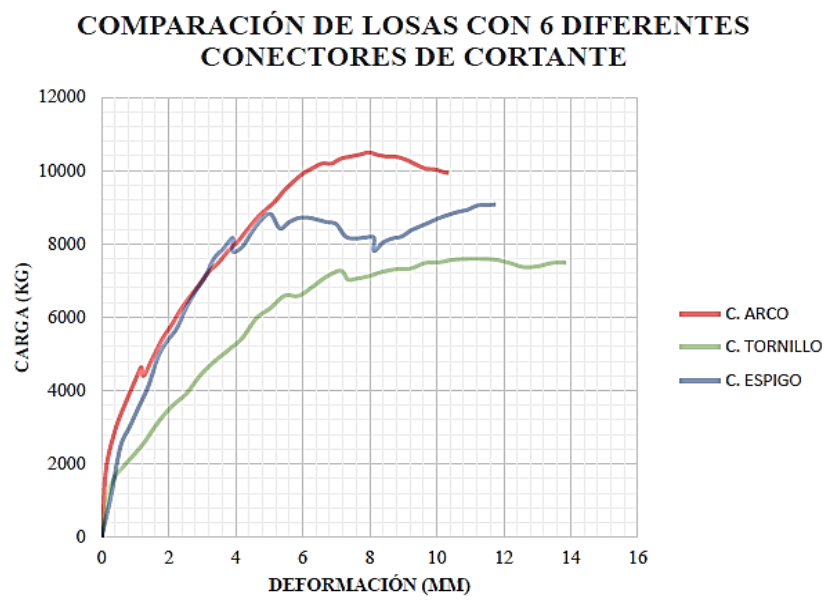

Figura 12 Carga vs Deformación para 3 tipos de conectores

Fuente: Barroso (2016).

Sin embargo, al incluir en la comparación al espécimen en el que los estribos actúan como conectores de corte, se observa que este último es el que mejor se desempeña, al alcanzar la mayor carga de rotura y deflexión moderada (figura13).

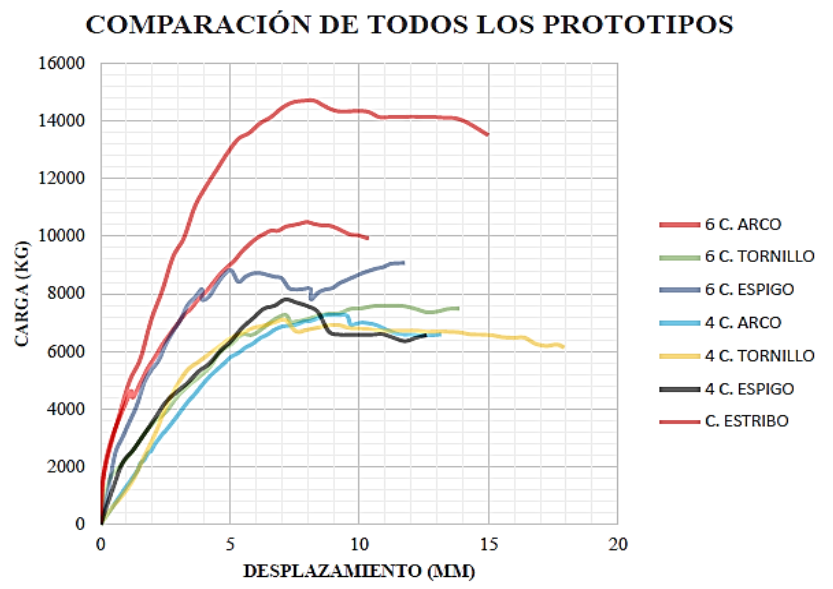

Figura 13 Carga vs Deformación todos los tipos de conectores Fuente: Barroso (2016).

\subsection{Trabajo como sección monolítica}

Adicionalmente para garantizar que la losa y la viga de hormigón trabajan como una sección monolítica, las secciones ensayadas deben alcanzar el momento teórico calculado con la ecuación (5) el cual equivale a $2321 \mathrm{~kg}^{*} \mathrm{~m}$, a excepción de la última muestra en la cual el peralte total de la sección disminuye en razón de que la losa se funde con la viga y no sobre ella, en este caso el momento nominal equivale a $1636 \mathrm{~kg}^{\star} \mathrm{m}$. 
Dado que el ensayo se realizó aplicando una carga puntual en el centro del claro, según la norma (ASTM C293-79, 2016), el momento resistente experimental se lo obtiene con la fórmula $P L / 4$ en la cual $P$ representa a la carga máxima a la rotura. En la tabla 2 se enlistan los prototipos ensayados junto con la carga de rotura, el momento resistente de la sección teórico y experimental.

Tabla 2 Momento Resistente de la Sección

\begin{tabular}{|l|l|l|l|}
\hline Muestra & $\begin{array}{l}\text { Carga } \\
\text { Máxima } \\
\mathbf{( K g )}\end{array}$ & $\begin{array}{l}\text { Momento } \\
\text { Teórico } \\
\left(\mathbf{K g}^{\star} \mathbf{m}\right)\end{array}$ & $\begin{array}{l}\text { Momento } \\
\text { Experimental } \\
\left(\mathbf{K g}^{\star} \mathbf{m}\right)\end{array}$ \\
\hline 1 (4 Arco) & 7285.71 & 2321 & 1876.071 \\
\hline 2 (6 Arco) & 10485.71 & 2321 & 2700.071 \\
\hline 3 (4 Tornillo) & 7114.29 & 2321 & 1831.929 \\
\hline 4 (6 Tornillo) & 7585.71 & 2321 & 1953.321 \\
\hline 5 (4 Espigo) & 7771.43 & 2321 & 2001.143 \\
\hline 6 (6 Espigo) & 9085.71 & 2321 & 2339.571 \\
\hline 7 (Estribo) & 14681.63 & 1636 & 3780.52 \\
\hline
\end{tabular}

Como se puede observar los conectores que garantizan el trabajo como sección compuesta son los tipos arco y espigo al utilizarse 6 conectores ( 2 por cada valle). Adicionalmente como se mencionó anteriormente la muestra fundida monolíticamente definitivamente garantiza el trabajo como sección compuesta, e incluso se logra un incremento del $130 \%$ del momento calculado teóricamente.

\subsection{Tipos de falla}

\subsubsection{Adherencia}

La primera falla presentada en las secciones analizadas (4 y 6 conectores) se produjo por la pérdida de adherencia entre la placa de acero deck y el hormigón en donde se observó una la separación entre el hormigón y la placa. Definitivamente, la carga a la que inicia la separación entre la placa y el hormigón depende directamente del número de conectores, lográndose una mayor carga en los prototipos elaborados con 6 conectores de corte. En la figura 14 se aprecia la falla por adherencia del prototipo elaborado con 6 conectores tipo arco. 


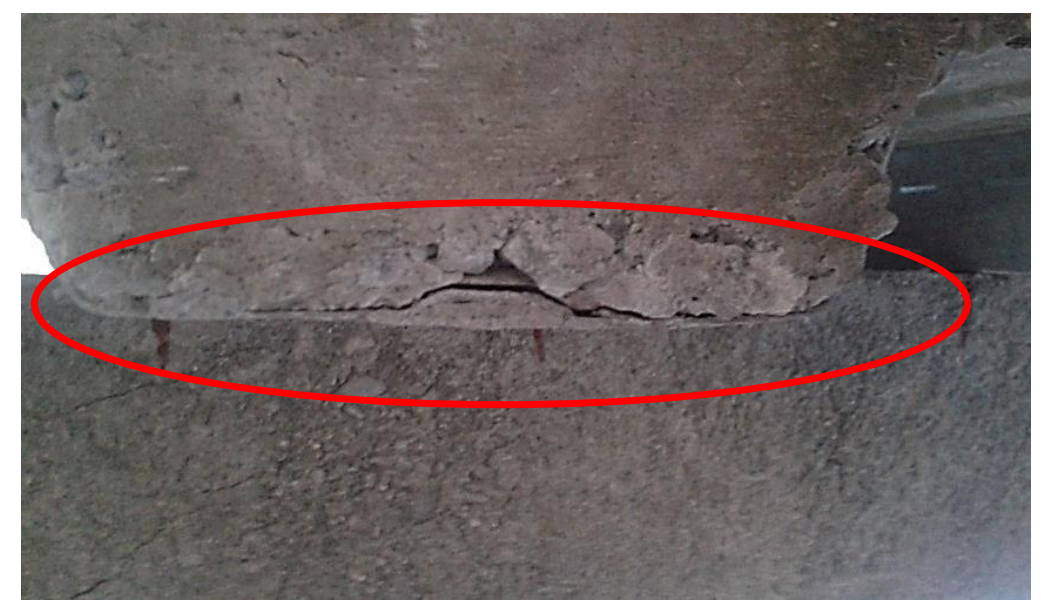

Figura 14 Separación entre la placa deck y la viga de hormigón Fuente: Barroso (2016).

\subsubsection{Desplazamiento entre la Losa Compuesta y la Viga de Hormigón}

Al final del ensayo se observó el desplazamiento horizontal entre la losa compuesta y la viga de hormigón que se identificó marcando la posición inicial en donde se apoyaba la placa sobre la viga y la posición en donde se encontraba al final del ensayo así se medió la distancia de desplazamiento que en el prototipo más crítico fue de $1.5 \mathrm{~cm}$. Cabe recalcar que para el caso del especímen 7 no existió desplazamiento horizontal, denotándose que es el mejor tipo de conector ante esta falla.

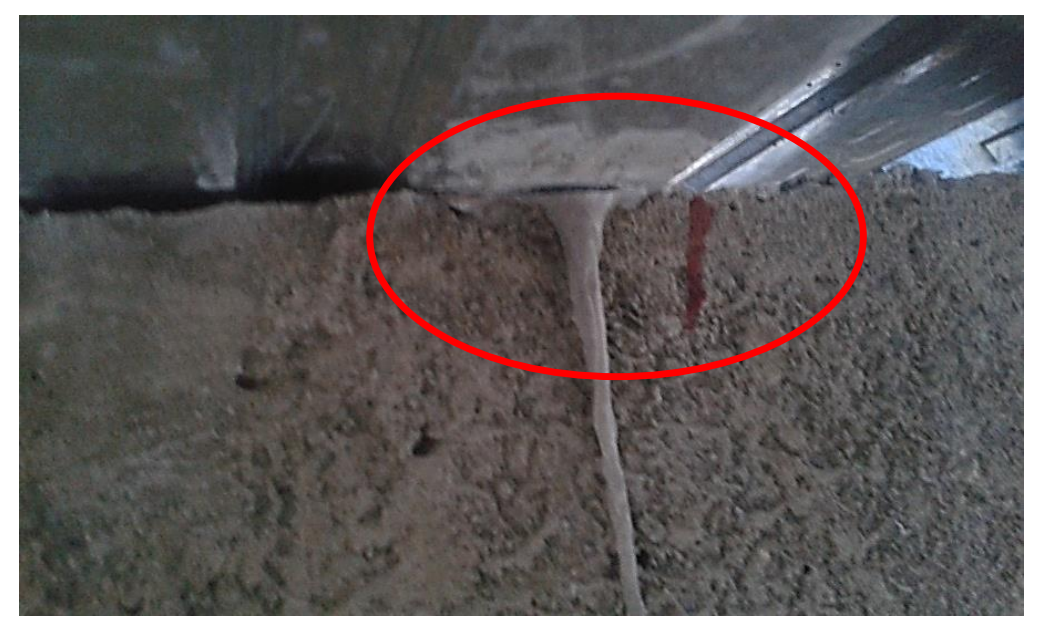

Figura 15 Falla por Deslizamiento

Fuente: Barroso (2016) 


\subsubsection{Falla en la viga de hormigón}

Finalmente, el último tipo de falla que se presentó en los prototipos y aquel que marcó la carga de rotura en la sección transversal, fue la falla a corte de la viga de hormigón. Es así como, los conectores de corte más efectivos son aquellos en los que se desarrolla una mejor transferencia de esfuerzos, evidenciada en una mayor carga de falla por corte en la viga de hormigón.

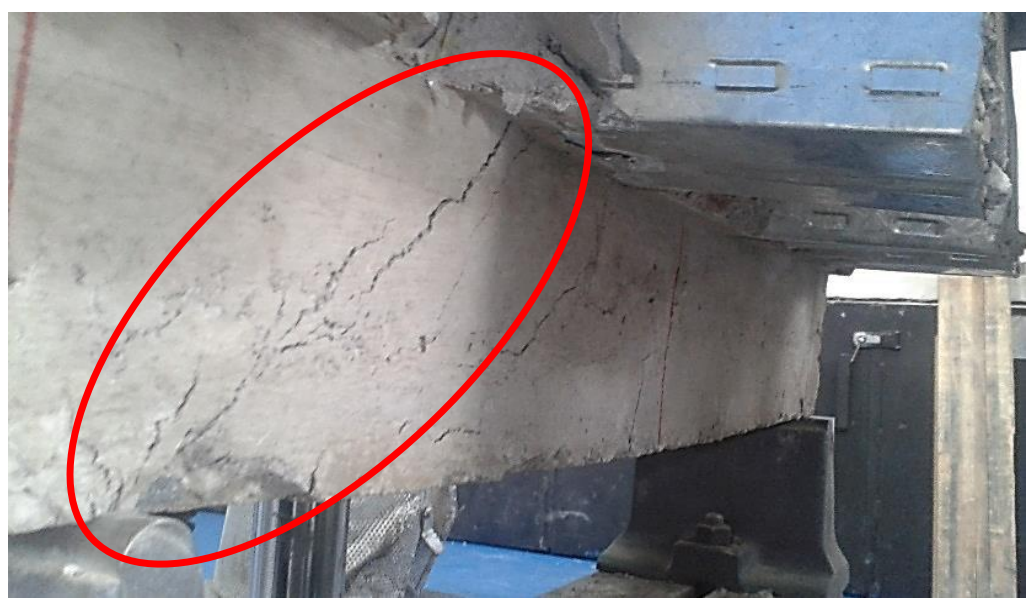

Figura 16 Falla a corte de la viga Fuente: Barroso (2016)

\section{CONCLUSIONES}

Las primeras fallas presentadas en las muestras se dieron en la viga de hormigón armado presentándose fallas a corte y a flexión en todas las muestras ensayadas, además de las fisuras presentas cerca de los conectores de cortante por concentración de esfuerzos.

En la sección fundida monolíticamente se observó mayor capacidad de carga ya que se presentó un deslizamiento máximo entre la viga y la losa de $4 \mathrm{~mm}$ al momento en que la viga presentó la falla.

Los conectores de cortante tipo arco, tornillo, y espigo no fueron capaces de impedir el deslizamiento entre la losa compuesta y la viga de hormigón, pero si el desprendimiento vertical de la sección compuesta.

Se determinó a través de los diagramas de carga vs deformación de cada prototipo que el modelo fundido monolíticamente con los estribos de las vigas, fue el que mejor desempeño alcanzó durante el ensayo ya que garantizó el comportamiento como un solo elemento al alcanzar un valor mayor al momento teórico calculado.

La separación y el número de conectores de cortante por valle de la placa colaborante, influye en el comportamiento de todo el elemento, por lo que se 
concluye que existe mayor capacidad de carga en los prototipos de seis conectores de cortante que en los de cuatro.

De los prototipos a ensayarse se determina que los únicos elementos que trabajan como una sola sección compuesta por la losa deck y la viga de hormigón son la muestra No.2 (seis conectores de cortante tipo arco de varilla corrugada de $\varnothing 8 \mathrm{~mm}$ ), $\mathrm{N}^{\circ} 6$ (seis conectores de cortante tipo perno) y $\mathrm{N}^{\circ} 7$ (conector tipo estribo) ya que se llegó al momento esperado teóricamente.

\section{REFERENCIAS}

1. Acero Deck. (2003). Manual Técnico para el uso de Placas Colaborantes para Losas de Entrepiso. Lima: Acero Deck.

2. ACl-318. (2011). Requisitos de Reglamento para Concreto Estructural y Comentario. Faminton Hills: American Concrete Institute.

3. AISC 360-10. (2010). Specification for Structural Steel Buildings. Chicago: American Institute of Steel Construction.

4. ASTM C293-79. (2016). Standard Test Method for Flexural Strength of Concrete (Using Simple Beam With Center-Point Loading). PA: American Society of Testing and Materials.

5. Barroso, M. (2016). Análisis de la influencia de los conectores de cortante en la unión viga de hormigón y losa compuesta. Tesis de Grado. Ambato: Universidad Técnica de Ambato.

6. Bonilla, J., Bezerra, L., Larrúa, R., Recarey, C., \& Mirambell, E. (2015). Modelación numérica con validación experimental aplicada al estudio del comportamiento de conectores tipo perno de estructuras compuestas de hormigón y acero. Revista Ingeniería de Construcción RIC, 30(1), 53-68.

7. Chávez Juan. (2014). Ensayo experimental a momento negativo de losas tipo deck en dirección perpendicular a los valles con diferente cuantía de acero de refuerzo. Tesis de Grado. Quito: Escuela Politécnica Nacional.

8. Hurtado, X., Molina, M., \& Linero, D. (2008). Comportamiento de conectores de cortante tipo tornillo de resistencia grado dos para un sistema de sección compuesta. Revista Ingeniería e Investigación, 28(2), 4-14.

9. McCormack, J., \& Csernak, S. (2012). Diseño de Estructuras de Acero (5ta Edición ed.). México: Alfaomega.

10. Ollgaard, J., Slutter, R., \& Fisher, J. (1971). Shear strength of stud connectors in lightweight and normal weight concrete. AISC Engineering Journal, 1(2010), 71-10. 
11. Ya , S., \& Yamada, K. (2008). Fatigue durability evaluation of trough to deck plate welded joint of orthotropic steel deck. Doboku Gakkai Ronbunshuu, 64(3), 603-616.

12. Zamora, A., \& Molina, M. (2008). Comportamiento de vigas de sección compuesta con perfiles de acero laminado y losa de concreto empleando conectores de cortante tipo tornillo grado dos. Revista Ingeniería e Investigación, 28(3), 11-21. 